\title{
TOTAL INTERNAL REFLECTION WITH FLUORESCENCE CORRELATION SPECTROSCOPY: APPLICATIONS TO SUBSTRATE-SUPPORTED PLANAR MEMBRANES
}

\author{
Nancy L. Thompson ${ }^{1,2}$, Xiang Wang ${ }^{2}$, and Punya Navaratnarajah ${ }^{3}$ \\ ${ }^{2}$ Department of Chemistry, University of North Carolina at Chapel Hill, Chapel Hill, North Carolina, \\ USA \\ ${ }^{3}$ Department of Biochemistry and Biophysics University of North Carolina at Chapel Hill, Chapel Hill, \\ North Carolina, USA
}

\begin{abstract}
In this review paper, the conceptual basis and experimental design of total internal reflection with fluorescence correlation spectroscopy (TIR-FCS) is described. The few applications to date of TIRFCS to supported membranes are discussed, in addition to a variety of applications not directly involving supported membranes. Methods related, but not technically equivalent, to TIR-FCS are also summarized. Future directions for TIR-FCS are outlined.
\end{abstract}

\section{Keywords}

Fluorescence Microscopy; Optical Microscopy; Evanescent; Fluctuation; Single Molecule; Surface Binding; Diffusion; Supported Membranes; Cell Membranes

\section{INTRODUCTION}

\begin{abstract}
In total internal reflection fluorescence microscopy (TIRFM), the excitation light is the surfaceassociated evanescent field created by internally reflecting a light source at a planar interface between two media with different refractive indices. Because the excitation light for fluorescence is confined to a small, sub-microscopic depth in the lower refractive index side of the interface, TIRFM is a surface-selective method and therefore, in many contexts, particularly amenable to investigations employing substrate-supported planar membranes. Many TIRFM methods are now well-established; others are in development. Some of the more well-developed TIRFM methods applicable to supported membranes include the use of evanescent illumination to measure surface densities of bound, fluorescent molecules for the purpose of measuring surface binding isotherms; to monitor fluorescence recovery after photobleaching for the purpose of examining the kinetics of surface association and dissociation, including surface rebinding events; and to examine the sensitivity of the steady-
\end{abstract}

(C) 2009 Elsevier Inc. All rights reserved.

1Corresponding Author Address: Department of Chemistry, Campus Box 3290, University of North Carolina at Chapel Hill, Chapel Hill, NC, 27599-3290, Telephone: (919) 962-0328, Fax: (919) 843-1580, nlt@unc.edu.

Publisher's Disclaimer: This is a PDF file of an unedited manuscript that has been accepted for publication. As a service to our customers we are providing this early version of the manuscript. The manuscript will undergo copyediting, typesetting, and review of the resulting proof before it is published in its final citable form. Please note that during the production process errors may be discovered which could affect the content, and all legal disclaimers that apply to the journal pertain. 
state fluorescence intensity to the evanescent wave polarization for the purpose of measuring order parameters for molecules in or on supported membranes. TIRFM is not solely applicable to supported membranes. This method has also been used in a variety of other contexts, including live cell biology, optical biosensors and polymer chemistry. For descriptions of TIRFM, the reader is referred to a number of recent reviews (Jaiswal and Simon, 2007; Lagerholm et al., 2005; Thompson and Pero, 2005; Schneckenburger, 2005; Axelrod, 2003; Steyer and Almers, 2001).

The method of fluorescence correlation spectroscopy (FCS) also encompasses both mature variants and variants under development applicable to a wide range of questions in membrane biophysics and other fields. In FCS, temporal fluctuations in the fluorescence measured from a small volume are analyzed to obtain information about the processes giving rise to the observed fluctuations. In most if not all FCS measurements, a requirement exists that only a small number of fluorescent molecules are present, on the average, in the observed volume. This requirement follows directly from the fact that the fluorescence fluctuations that FCS monitors are usually number fluctuations; i.e., fluctuations in the number of fluorescent molecules in the observed volume. Thus, FCS and supported membranes are similar in that both methods require only very small amounts of material. The most commonly used version of FCS remains its historical one in which the method is used to measure translational mobility, but other types of FCS are also now well-developed and mature. These methods include, but are not limited to, two-color fluorescence fluctuation cross-correlation for quantifying bimolecular binding, photon counting histograms for characterizing molecular aggregation, and spatial fluorescence fluctuation analysis for detecting molecular clustering. FCS is most commonly carried out with confocal optics. In some of these cases, evanescent illumination combined with limited detection from the sample plane might be a superior approach because of the higher axial resolution (e.g., measurement of membrane dynamics in the presence of soluble fluorophores or high cytosolic autofluorescence). FCS has been described in a number of recent reviews as well as a book (Haustein and Schwille, 2007; Blom et al., 2006; Vukojevic et al., 2005; Gosch and Rigler, 2005; Enderlein et al., 2004; Muller et al., 2003; Thompson et al., 2002; Rigler and Elson, 2001).

In this paper, the emerging technique of combining evanescent illumination with fluorescence correlation spectroscopy (TIR-FCS) is reviewed, with emphasis placed primarily, but not exclusively, on applications to supported membranes. Methods related to TIR-FCS as well as potential future developments in TIR-FCS are also discussed. The combination of TIRFM and FCS has been previously reviewed (Thompson and Pero, 2006; Anhut et al., 2005) and also briefly discussed in context of similar techniques (Ries and Schwille, 2008; Groves et al., 2008; Garciá-Sáez and Schwille, 2007; Blom et al., 2006). An additional paper that discusses in depth the experimental details of TIR-FCS, including many of the current pitfalls and limitations, has also been published recently (Thompson and Steele, 2007). Review papers describing methods for constructing supported membranes and evaluating molecular behavior on or near the membranes are also available (Kiessling et al., 2009; Chan and Boxer, 2007; Castellana and Cremer, 2006).

\section{CONCEPTUAL BASIS AND EXPERIMENTAL DESIGN}

The conceptual basis of the simplest form of TIR-FCS was theoretically suggested and shortly thereafter experimentally demonstrated many years ago (Thompson et al., 1981; Thompson and Axelrod, 1983). This early notion was to use the evanescent wave generated by total internal reflection along the optical axis of a microscope (here, denoted as the z-axis) along with observation restriction in the sample plane (here, denoted as the $\mathrm{x}-\mathrm{y}$ plane) to create a very small, surface-selective observation volume from which fluorescence fluctuations could be monitored. The goal was to autocorrelate the observed fluorescence fluctuations for the 
purpose of obtaining information about the association and dissociation kinetics of fluorescent molecules in solution reversibly binding to surface sites, and the lateral mobility of the surface binding sites. Subsequently, TIR-FCS has evolved considerably although new variants are still being developed.

The key requirements of a successful TIR-FCS apparatus based on number fluctuations are somewhat simple as compared to many types of fluorescence microscopy. The average number of fluorescent molecules in the observed volume must be small enough so that the number fluctuations are large enough to give correlations with large enough magnitudes. Most FCS measurements are carried out with $\leq 100$ fluorescent molecules in the observed volume. At higher molecular numbers, the fluorescence fluctuations of interest are often dominated by background signals or fluctuations arising from mechanical instabilities. This requirement for having a small number of fluorescent molecules in the volume of interest demands a balance between a small observation volume and a low concentration of fluorescent molecules residing, on the average, in the observed volume. Signal-to-noise requirements necessitate a high emission and detection efficiency of the observed fluorescence. Because the method depends on accurately measuring correlated fluctuations from the fluorescent molecules of interest, background light cannot be neglected.

As described by elementary and idealized optical theory, total internal reflection occurs when a plane wave traveling in a higher refractive index medium encounters a planar interface with a lower refractive index medium at an angle $\alpha$, defined from the normal to the interface, greater than the "critical angle", $\alpha_{c}=\sin ^{-1}\left(n_{2} / n_{1}\right)$, where $n_{2}$ is the lower refractive index and $n_{1}$ is the higher refractive index. When internal reflection occurs, the plane wave completely reflects into the higher refractive index medium but also creates a surface-associated "evanescent" field in the lower refractive index medium. The evanescent field propagates parallel to the interface and the evanescent intensity penetrates into the lower refractive index medium with an idealized exponential form having a characteristic decay length $\mathrm{d}=\lambda_{0} /\left[4 \pi\left(\mathrm{n}_{1}^{2} \sin ^{2} \alpha-\right.\right.$ $\left.\mathrm{n}_{2}^{2}\right)^{1 / 2}$, where $\lambda_{0}$ is the vacuum wavelength of the incident light. Typical values for these parameters are $\lambda_{0} \approx 500 \mathrm{~nm}$ (visible light), $\mathrm{n}_{1} \approx 1.5$ (glass or fused silica), $\mathrm{n}_{2} \approx 1.33$ (water), $\alpha \approx 75^{\circ}, \alpha_{\mathrm{c}} \approx 65^{\circ}$, and $\mathrm{d} \approx 70 \mathrm{~nm}$.

The evanescent intensity at the interface depends on the two refractive indices and the incidence angle (Thompson et al., 1993; Axelrod et al., 1984). For typical conditions, this maximum evanescent intensity is not equivalent to, but is on the order of, the intensity of the incident light. The polarization of the electric field is somewhat unusual for evanescent waves as compared to freely propagating plane waves. In this work, the incidence plane is denoted as the $\mathrm{x}-\mathrm{z}$ plane, the optical axis of the microscope is denoted by $\mathrm{z}$, and the distance into the lower refractive index medium is denoted by $z>0$. When an incident plane wave, propagating in the $\mathrm{x}-\mathrm{z}$ plane, is polarized perpendicular to the incidence plane (s-polarized, along the $\mathrm{y}$-axis), the evanescent electric field is also polarized perpendicular to the incidence plane (along the $y-$ axis). However, an atypical evanescent polarization is generated when the incident light is polarized in the incidence plane (p-polarized, in the x-z plane). In this case, the evanescent electric field contains components both in line with the direction of evanescent field propagation (the $\mathrm{x}$-axis) and perpendicular to this direction (along the $\mathrm{z}$-axis). As compared to conventional epi-illumination, this property of evanescent fields produced with p-polarized light allows measurement of order parameters with respect to the polar angle from the interface normal (Thompson et al., 1984). These measurements are particularly useful for monitoring the quality of supported membranes.

The theory above describes the behavior of a single plane wave. For a more accurate description of the intensity and polarization characteristics of an evanescent wave generated by a given optical apparatus, the problem can be approached by expressing the excitation light source as 
an integral over plane waves, transforming the behavior of individual plane waves through components of the excitation path, and summing the result, as shown in an early example (Burghardt and Thompson, 1984). The properties of evanescent fields both in theory and in the context of different experimental apparatuses have been discussed in extensive detail (e.g., Burghardt et al., 2006b; Mattheyses and Axelrod, 2006; Anhut et al., 2005; Girard et al., 2000; Knoll, 1998; Huang and Thompson, 1993; Hellen and Axelrod, 1987; Agudin and Platzeck, 1978).

Several types of experimental arrangements have been used for TIR-FCS (Thompson and Steele, 2007). Both through-prism and through-objective mechanisms for generating evanescent waves at the sample plane have been employed (Figure 1). Two advantages of through-prism TIRFM are the versatility of optical alignment, and a lower background arising from the fact that the incident beam does not enter the interior of the microscope. This geometry is also readily amenable to constructing evanescent interference patterns (Huang et al., 1994) and to the use of very high refractive index substrates (Starr and Thompson, 2000). Advantages of through-objective TIRFM include sample-top accessibility, although through-prism configurations allowing such accessibility have also been designed (Axelrod, 2001); decreased stray laser light in regions external to the microscope; and, often, better image quality. In addition, the effect of the planar dielectric interface on the angular emission profile of nearby fluorophores is such that more fluorescence is emitted through the higher refractive index side of the interface compared to the lower refractive index side (for fluorophores close to the interface) and the effective evanescent wave depth is also somewhat smaller (Steyer and Almers, 2001).

In most cases, the size of the evanescent illumination in the sample plane is much larger than the area from which fluorescence is detected to achieve the requirement that the observation volume be very small. Methods for restricting fluorescence collection to a small area in the $\mathrm{x}$ y sample plane (typically, $\sim 1 \mathrm{~m}^{2}$ ) include using a pinhole (Starr and Thompson, 2002; Lieto et al., 2003) or a fiber optic (Hassler et al., 2005a,b) placed at a back image plane of the microscope. Nearly all TIR-FCS instruments use high aperture objectives for high fluorescence collection efficiencies (Leutenegger and Lasser, 2008). Single-point fluorescence detection has been carried out both with highly sensitive single-photon counting photomultipliers (Pero et al., 2006) or silicon avalanche diodes (Leutenegger et al., 2006). The use of fast EMCCD cameras for multi-point fluorescence detection is rapidly emerging (Kannan et al., 2007).

Because a small number of fluorescent molecules are within the observed volume, the measured fluorescence fluctuates significantly with time as individual fluorescent molecules diffuse in and out of the volume, and, in some cases, bind to and dissociate from sites on the surface (Figure 2). In the simplest case, the fluorescence fluctuations are autocorrelated as

$$
G(\tau)=\frac{<\delta F(t) \delta F(t+\tau)>}{<F>^{2}}
$$

where $F(t)$ is the time-dependent fluorescence at time $t,\langle F\rangle$ is the time-averaged fluorescence, and $\delta \mathrm{F}(\mathrm{t})$ is the time-dependent fluctuation of the fluorescence from its average value. $\mathrm{G}(\tau)$ typically decays to zero when the lag-time $\tau$ approaches infinity. The magnitude of this function contains information about the average number of fluorescent molecules in the observed volume. The rate and shape of the time decay of $\mathrm{G}(\tau)$ contain information about the processes giving rise to the fluorescence fluctuations; for example, diffusion coefficients in solution, association and dissociation rate constants for the surface binding sites, enzyme kinetics, and/ or spectroscopic dynamics (e.g., triplet state kinetics or blinking). 
A key aspect to a successful TIR-FCS study is the signal-to-noise ratio of the correlaton function. Starting with the initial work of Koppel (1974), several theoretical treatments of this question have been carried out (Kolin et al., 2006; Costantino et al., 2005; Saffarian and Elson, 2003; Wohland et al., 2001; Meseth et al., 1999; Kask et al., 1997; Qian, 1990) although none of these works specifically addresses TIR-FCS. The signal-to-noise ratio generally increases with the number of photons collected per molecule per sample time and the total time over which fluctuations are correlated.

Many studies use TIRFM solely to reduce background without making use of the evanescent field directly, particularly those that monitor single fluorescent molecules. Evanescent illumination does indeed often result in a lower overall background signal (Huang and Thompson, 1996). However, even background light that is not correlated with the measured fluorescence affects the magnitude of fluorescence fluctuation correlation functions and appropriate corrections must be made (Thompson and Axelod, 1983). The primary sources of background in through-prism TIRFM are usually scattered evanescent light and substrate luminescence (which can be significantly reduced by using fused sillica rather than glass). In through-objective TIRFM, the excitation light that passes through the interior of the microscope also contributes to the background. Calculations demonstrating methods of correcting for uncorrelated background signals (Thompson, 1991) are readily generalized even to correlated background signals.

Nearly all FCS measurements rely on theoretical models for the shape of the correlation functions to extract the parameters of interest from the magnitude and shape of these data. Consquently, a body of theoretical work has been developed for the purpose of interpreting measured TIR-FCS correlation functions. These theories are specific both to the processes giving rise to the fluorescence fluctuations (e.g., diffusion through the evanescent wave, surface binding and dissociation kinetics, enzyme kinetics, triple state effects) and to the particular optical arrangement that is employed (Ries et al., 2008a; Kyoung and Sheets, 2008; Hassler et al., 2007; Borejdo et al., 2006; Leutenegger et al., 2006; Pero et al., 2006; Hassler et al., 2005a,b; Holt et al., 2004; Lieto and Thompson, 2004; Starr and Thompson, 2001; Johns et al., 2001; Widengren et al., 1995; Thompson, 1982; Thompson et al., 1981).

\section{APPLICATIONS}

\section{Molecular Mobility Close to Surfaces}

The simplest application of TIR-FCS is to autocorrelate fluorescence fluctuations arising from small fluorophores diffusing through the depth of the evanescent wave. This type of measurement has been used primarily to demonstrate that the method is working properly (e.g., Leutenegger et al., 2006; Ohsugi et al., 2006; Ohsugi and Kinjo, 2006; Anhut et al., 2005; Hassler et al., 2005a,b; Harlepp et al., 2004; Ruckstuhl and Seeger, 2004). In addition, given an estimate of the evanescent wave depth $\mathrm{d}, \mathrm{G}(\tau)$ provides an estimate of the diffusion coefficient in solution; alternatively, given a known diffusion coefficient, $G(\tau)$ provides an estimate of the evanescent wave depth. This type of TIR-FCS study must be carried out under conditions for which the fluorescent molecules do not bind to the surface. Thus, supported membranes are useful in this context because they are particularly resistant to nonspecific binding. Also, because the diffusional decay rate is fast for small fluorophores, the autocorrelation function must account for triplet state contributions.

TIR-FCS has also been used to measure the concentrations and diffusion coefficients of fluorescently labeled IgG in close proximity to supported membranes (Figure 3) (Starr and Thompson, 2002). These measurements were carried out for membranes with different lipid compositions and surface charges and for solutions of varying $\mathrm{pH}$ and ionic strength. The purpose was to determine if previously observed non-idealities associated with the kinetics of 
specific ligand-receptor interactions at membranes might be related to local deviations in the ligand concentration or mobility very near membrane surfaces. The results, for an evanescent wave depth of approximately $70 \mathrm{~nm}$, showed no statistically significant change in the local $\mathrm{IgG}$ concentration for the numerous sample conditions explored, and no change in the local IgG mobility for membranes with different charges or for solutions with different $\mathrm{pH}$ values spanning the IgG isoelectric point. However, a significant decrease in the local mobility was detected with increasing ionic strength. This result suggested that strong hydrodynamic interactions occur between proteins and membrane surfaces and that these interactions are amplified by increasing ionic strength. To test this hypothesis, TIR-FCS was used to measure, close to supported membrane surfaces, the diffusion coefficients of fluorescently-labeled antibodies, antibody fragments and antibody complexes with a range of hydrodynamic radii (Pero et al., 2006). It was found that planar lipid bilayers reduced the diffusion coefficients of these molecules in a size dependent manner. Specifically, diffusion coefficients close to membranes were seen to decrease with hydrodynamic radii above what would be predicted in solution and in agreement with predictions of hydrodynamic theories addressing spherical particles diffusing close to planar walls. The conclusion was that hydrodynamic effects are the primary factor governing the mobility of proteins near phospholipid bilayer surfaces.

Molecular transport in sol-gel films has been characterized by using TIR-FCS (McCain and Harris, 2003). The translational mobilities of rhodamine $6 \mathrm{G}$ dye molecules inside sol-gel films made by dip-coating porous silica particles onto microscope slides were measured from the time-decay of TIR-FCS autocorrelation functions. These measurements defined the tortuosities for films of differing thicknesses, both before and after ethanol annealing. A subsequent paper addressed the question of interparticle vs. intraparticle molecular mobilities in these sol-gel films (McCain et al., 2004a). Fluorescent probes of varying size were created as polyamidoamine dendrimers labeled with carboxyrhodamine 6G. Fluorescently labeled dendrimers were chosen as probes because they are available in monodisperse sizes ranging from 3-20 nm, thus making them amenable to the exploration of pore sizes within the films. By using TIR-FCS, it was found that the dendrimers nonspecifically adsorbed to bare silica surfaces, a property that would significantly complicate their use as probes of molecular transport in the sol-gel films. To circumvent this difficulty, the positively charged primary amine groups on the dendrimers were covalently modified to negatively charged carboxylic acid groups. This modification significantly reduced dendrimer adsorption, presumably because the silica surfaces are negatively charged. The carboxylated dendrimers were then placed inside sol-gel films and the mobilities were very carefully examined using TIR-FCS with the goal of understanding intraparticle vs. interparticle mobility as well as tortuosities (McCain et al., 2004b).

\section{Mobility of Particles Close to Surfaces}

TIR-FCS mobility measurements have also been carried out for fluorescent particles rather than molecules close to membrane surfaces. The very first demonstration of TIR-FCS as a viable method, to the knowledge of the authors, was one in which the diffusion of fluorescentlylabeled viruses through the evanescent wave was proposed as a method of viral identification (Hirschfeld and Block, 1977; Hirschfeld et al., 1977). Although outdated as a method for viral identification, these studies are mentioned because they foresaw the potential of TIR-FCS as a biophysical method for monitoring particle diffusion close to surfaces.

A very recent work has examined the diffusive behavior of phospholipid vesicles very close to supported membrane surfaces by using TIR-FCS, with an emphasis on understanding factors leading to vesicle-membrane fusion (Kyoung and Sheets, 2008). In this work, the local vesicle mobility was examined for a wide range of conditions including different ionic strengths, different solution $\mathrm{pH}$ values, and different lipid compositions. The results provide a 
comprehensive view of the primary factors governing vesicle motions very close to membrane surfaces.

TIR-FCS has also been used to monitor the mobility of intracellular vesicles close to the basal membranes of live, adherent cells. In one study, the motions of secretory granules in the cytoplasms of adherent bovine chromaffin cells near their plasma membranes were examined in detail by using various TIRFM approaches (Johns et al., 2001). Secretory granules were labeled with a GFP conjugate of pro-atrial natriuretic peptide, and the behavior of the highly fluorescent granules was monitored before and during exocytosis. As part of this comprehensive work, the granule velocities, as measured with evanescent excitation and sequential imaging, were autocorrelated to determine if granule motion was Brownian in nature or if the autocorrelation data showed evidence of possible granule caging or tethering. The data, quite interestingly, were indicative of the latter situation in some cases. In a related study, TIR-FCS was used to monitor the mobilities of fluorescently labeled synaptic vesicles near ribbon synapses in retinal bipolar cells (Holt et al., 2004).

\section{Non-Specific, Reversible Surface Association and Dissociation Kinetics}

TIR-FCS can be employed to examine not only mobility close to an evanescently excited surface but also to examine reversible surface association and dissociation kinetics. The concept governing this idea is that $\mathrm{G}(\tau)$ will contain information directly related to the surface association and dissociation kinetic rate constants, given an assumed model for the mechanism of reversible surface binding. It is noted, however, that with TIR-FCS, it is possible to characterize diffusion close to surfaces in the absence of surface interactions, but it is not always possible to characterize surface interactions in the absence of diffusion towards and away from the surface of interest. In particular, if the characteristic times for surface association and dissociation are close enough to the characteristic times for diffusion through the evanescent wave, theoretical models must account for both processes.

The first class of this type of measurement belongs to one which can be classified as nonspecific in the sense that the fluorescent molecules in solution do not reversibly interact with specific, molecular surface sites on the surface but, rather, with diverse sites on the surface. This first class was the basis for one of the first experimental demonstration of TIR-FCS. In this work, the kinetics associated with nonspecific, reversible adsorption of rhodamine-labeled immunoglobulin and insulin to serum albumin-coated fused silica slides were examined (Thompson and Axelrod, 1983).

TIR-FCS was revived from a period of dormancy (roughly 1983-1998) in two studies motivated by the need to understand surface interactions in reversed-phase chromatograpy. In the first study, the small fluorescent molecule rhodamine 6G was used as a probe in water/ methanol solutions at bare silica surfaces and at silica surfaces derivatized with C-18 alkyl chains (Hansen and Harris, 1998a). In these measurements, the magnitude of the fluorescence fluctuation autocorrelation was inversely related to the average number of fluorescent molecules in the observed volume, and $\mathrm{G}(0)$ was thus used to obtain fluorophore surface densities. The measured surface densities were used to determine equilibrium constants for the reversible adsorption of rhodamine $6 \mathrm{G}$ to the chromatographic support mimics for a variety of solution conditions. A second study extended the work to measure dye adsorption and desorption kinetic rates (Hansen and Harris, 1998b).

TIR-FCS has recently been used to study the behavior of proteins near modified surfaces in the presence of a surfactant (Sonesson et al., 2008). The motivation for this work was that transient interactions of proteins with surfaces are important in a variety of fields including, for example, chromatography. The proteins examined were fluorescein-labeled bovine serum albumin (BSA) and fluorescein-labeled Thermomyces lanuginosus lipase (TLL); the solid 
support was $\mathrm{C}_{18}$-coated glass; and the nonionic/anionic detergent was a mixture of hexaethylene glycol dodecyl ether $\left(\mathrm{C}_{12} \mathrm{E}_{6}\right)$ and linear alkylbenzene sulfonate (LAS). The fluorescence fluctuation autocorrelation functions obtained from TIR-FCS measurements were analyzed in terms of previously developed theoretical models for surface interactions, free diffusion within the evanescent field, and photophysical kinetics. This work demonstrated that the binding of the fluorescently labeled proteins to the surface decreased when the surfactant concentration was increased. Furthermore, the surface interaction rate, which in this case was a function of the association and dissociation rate constants, as well as the concentration of fluorescently labeled proteins in solution, increased with increasing surfactant. Overall, the results implied that the average surface residency time of the proteins decreased as the surfactant concentration was increased.

\section{Specific, Reversible Surface Association and Dissociation Kinetics}

One way to use TIR-FCS to look directly at the kinetics of reversible, specific ligand-receptor interactions is to embed receptors in substrate-supported planar membranes and then allow fluorescently labeled ligands to freely diffuse, bind to, and dissociate from the surfaceassociated receptors (Figure 4). This approach has recently been used to examine the kinetics of fluorescently labeled IgG specifically and reversibly associating with the mouse Fc receptor Fc $\gamma$ RII, which was purified and reconstituted into supported membranes (Lieto et al., 2003). The experimental parameters required for successful implementation of this type of measurement are not completely straightforward. First, to ensure that a high enough fraction of the evanescently excited fluorescence arises from surface-bound fluorescent ligands, as opposed to those merely close to the surface, a high enough receptor density must be used. Second, large enough ligand concentrations must be used to avoid working far below the midpoint of the binding isotherm, where rare, tight, nonspecific binding sites might dominate the surface-bound species. However, these constraints can be contradictory to the mandate of FCS where a small number of observed, fluorescent molecules is required so that the magnitude of the fluorescence fluctuations relative to the mean fluorescence value is large enough to be accurately measured. The method used to circumvent this difficulty was to mix nonfluorescent IgG with a much smaller amount of fluorescently labeled IgG (Lieto and Thompson, 2004). As shown in Figure 3 and Figure 4, G( $\tau$ ) contained a long-time component when receptors were present that was not observed in the absence of receptors. Another key feature was that for identical concentrations of fluorescent IgG in solution, $\mathrm{G}(0)$ was lower rather than higher for membranes containing receptors; this result can be understood in the context that $G(0)$ is, roughly, inversely proportional to the average number of fluorescent molecules in the observed volume and that this average number is higher when surface-binding sites are present. Fitting the data to appropriate theoretical forms gave a measure of the dissociation kinetic rate consistent with that measured by other methods.

\section{Enzyme Kinetics}

TIR-FCS has also been used to study the kinetics associated with the catalytic cycle of horseradish peroxidase (HRP) (Hassler et al., 2007). To make single molecule observations, streptavidin-labeled HRP molecules were immobilized on microscope slides functionalized with poly(ethyleneglycol)biotin bound to poly(L-lysine) (PLL-PEG-biotin). TIR-FCS was then employed to follow the HRP-catalyzed reduction of a fluorogenic agent. The enzyme had two spectroscopically distinct states: a fluorescent enzyme-product (EP) complex, and all other states that were non-fluorescent. Images obtained from a CCD camera were used to isolate a single enzyme, and a single photon counting silicon avalanche diode was employed to acquire time-dependent fluorescence intensity data for the specific HRP molecule. The fluorescence data were autocorrelated, and fit to an appropriate functional form to obtain the rate constant for the formation of the EP complex. It was found that this rate constant was distributed over several orders of magnitude, suggesting that conformational heterogeneity (dynamic disorder) 
of individual enzymes is responsible for the different catalytic rates observed during ensemble measurements.

\section{Cross-Correlation TIR-FCS}

A mature type of solution FCS is one in which the fluorescence fluctuations from species labeled with different, spectrally resolvable fluorophores are cross-correlated, often with the intention of detecting bimolecular binding (Bacia et al., 2006; Bacia and Schwille, 2007; Widengren et al., 2001; Wallace et al., 2000; Schwille et al., 1997). This method has now been combined with through-objective internal reflection (Leutenegger et al., 2006). In this short, first demonstration, cross-correlation TIR-FCS was validated by examining dual-labeled, double-stranded DNA diffusing through the evanescent wave.

\section{Live Cell Applications}

TIR-FCS has been used to examine processes near the basal plasma membranes of surfaceadherent, intact cells (see above, Holt et al., 2004; Johns et al. 2001). In a more recent work, TIR-FCS was used to study the lateral mobility of transfected, farnesylated enhanced green fluorescent protein (F-EGFP) in or near the plasma membranes of COS7 and HeLa cells (Ohsugi et al., 2006). Through-objective total internal reflection was employed to create an evanescent field, illuminating a thin layer of the cells near glass surfaces to which they were adhered. The resulting fluorescence yielded autocorrelation functions that could be fit by a combined two- and three-dimensional diffusion model. The three dimensional diffusion component was on the order of $10^{-7} \mathrm{~cm}^{2} \mathrm{~s}^{-1}$, and was assigned to the mobility of free F-EGFP in the cytosol. The slower, two-dimensional diffusion component was on the order of $10^{-9}$ $\mathrm{cm}^{2} \mathrm{~s}^{-1}$, and was assigned to the lateral mobility of membrane-associated F-EGFP. These observations were confirmed with single molecule tracking and confocal FCS.

In a related study, TIR-FCS was used to follow the motions of an intracellular EGFP-labeled protein kinase $\mathrm{C}$ (PKC $\beta \mathrm{II}$ ) in or near the membranes of adherent HeLa cells (Ohsugi and Kinjo, 2006). Again, components were detected in the measured autocorrelation functions thought to represent both fast $\left(\sim 10^{-7} \mathrm{~cm}^{2} \mathrm{~s}^{-1}\right)$, three-dimensional mobility in the cytosol and slow $\left(\sim 10^{-9} \mathrm{~cm}^{2} \mathrm{~s}^{-1}\right)$, two-dimensional mobility in the basal membrane. After stimulation with ATP, the fraction of PKC $\beta I I$ associated with the membrane increased.

In another cellular application, a variation of TIR-FCS was used to study paxillin dynamics in focal adhesions of migratory cells (Digman et al., 2008). CHO cells expressing the fusion protein paxillin-EGFP were plated on glass-bottom dishes pre-coated with fibronectin. To determine the spatial and temporal dependence of paxillin dynamics in motile cells, an EMCCD camera was employed to collect high resolution images of the cells, following TIR illumination. As the evanescent wave depth was about $100 \mathrm{~nm}$, only those EGFP-labeled paxillins close to the plasma membrane and, more importantly, near focal adhesions were visualized.

Furthermore, the image acquisition rate of 90 frames s ${ }^{-1}$ also meant that diffusion of paxillin in the cytosol was too fast to be detected. This ensured that the observed fluorescence fluctuations reflected binding interactions of EGFP-paxillin at focal adhesions. With an eye towards quantifying the spatial dependence of paxillin dynamics, fluorescence fluctuation autocorrelation functions were computed for each pixel in the collected images, and fit with a kinetic model for binding. These data yielded exchange rates that varied from $0.1 \mathrm{~s}^{-1}$ to 10 $\mathrm{s}^{-1}$. Both scanning FCS and TIR-FCS analyses showed that the fastest exchange rates were associated with the leading edge of the cell, whereas the slower rates corresponded to the trailing edge. This result suggested that the rate of paxillin incorporation during adhesion assembly at the leading edge was fast, while paxillin loss during adhesion disassembly at the trailing edge was slow. Furthermore, extrapolated $\mathrm{G}(0)$ and intensity values demonstrated that 
protein clusters containing many paxillin molecules dissociated during disassembly, but paxillin was incorporated at the leading edge as monomers.

\section{Fast Cameras}

The use of fast EMCCD cameras with FCS promises parallel detection (i.e., the acquisition of multiple autocorrelation functions by using a series of images). In this arrangement, the sample volume is restricted by the pixilation of the camera. This idea has recently been explored by examining the diffusion of rhodamine-labeled phospholipids in supported membranes as well as epidermal growth factor receptors tagged with fluorescent proteins and present in the membranes of CHO cells (Kannan et al., 2007). In this work, emphasis was placed on determining the degree of cross-talk among adjacent pixels and how one might accommodate that effect in the simplest case where the goal is to autocorrelate fluorescence fluctuations arising from areas defined by single or near-neighbor pixels without accounting for cross-talk between the different multiplexed observation areas. Another emphasis was on determining the minimum number of image frames required to achieve high enough signal-to-noise ratios in the autocorrelation functions.

\section{RELATED METHODS}

\section{Overview}

An observation volume containing a large number of fluorescently labeled species yields ensemble-averaged fluorescence intensities. As FCS relies on the stochastic behavior of individual molecules, fluorescence emanating from a large number of molecules will not lend itself to being effectively correlated, making it impossible to obtain informative fluorescence fluctuation autocorrelation curves. Therefore, FCS requires that the average number of fluorescently labeled species in the observation volume be small. This requirement can often be readily met in in vitro experiments by adjusting concentrations so that a small number of fluorescent molecules is present in the observation volume. In situ, however, molecular concentrations are fixed, and it is more difficult to limit the number of fluorescently labeled molecules being observed. An alternative way to pursue the observation of single fluorescent molecules or small numbers of fluorescent molecules is to further reduce the detection volume. In addition to the use of evanescent excitation, a number of related techniques have been successfully employed to limit the detection volume to a few attoL $\left(10^{-18} \mathrm{~L}\right.$ ) (for a review, see Blom et al., 2006). Furthermore, as many of these techniques limit detection volumes by means of axial confinement, they are particularly applicable to the study of molecular dynamics at or near supported planar membranes.

\section{Near-Field Scanning Optical Microscopy}

Near-field scanning optical microscopy (NSOM) can exceed diffraction-limited resolution by selectively exciting fluorescence from molecules within the evanescent wave associated with the tip. As the evanescent intensity decays with distance from the tip, NSOM requires that the tip be placed close to the surface. A piezoelectric stage is used to scan the surface of the sample. Recently, NSOM, employing a cantilevered optical fiber probe, was used in combination with FCS to monitor the diffusion of rhodamine $6 \mathrm{G}$ at a concentration of $100 \mathrm{nM}$ (Lewis et al., 2007). This concentration is approximately ten times larger than that typically used in FCS experiments with confocal illumination.

\section{Focused TIRFM}

Recent work has demonstrated that focused TIRFM (fTIRFM) can be used to reduce observation volumes. In one approach, a parabolic mirror objective (PMO) was used to create focused evanescent illumination in the sample plane (Ruckstuhl and Seeger, 2003). Subsequent 
measurements demonstrated that fTIRFM, when combined with confocal detection, yielded observation volumes less than 5 attoL (Ruckstuhl and Seeger, 2004). A similar method used only a conventional high aperture objective, associated optics, and a confocal pinhole to generate detection volumes as small as 3 attoL (Burghardt et al., 2006b). This apparatus enabled in situ examinations of intact skeletal muscle fibers in which the detection volume isolated approximately five fluorescently labeled myosin cross-bridges.

\section{Supercritical Angle Fluorescence}

Supercritical angle fluorescence (SAF) detection is another technique that can be used to reduce observation volumes. When a fluorescent molecule is close to a planar dielectric interface, the near-field of the emitted radiation couples to the interface and a significant fraction of the fluorescence propagates as far-field radiation into the high refractive index side of the interface, at angles greater than the critical angle. Therefore, by restricting fluorescence detection to supercritical angles, one can achieve axial confinement and reduce detection volumes. In addition to limiting the lateral extent of the observation volume in objective-type fTIRFM, PMOs also enhance axial confinement because they can be made to be selective for fluorescence emitted at supercritical angles (Ruckstuhl and Seeger, 2003; Ruckstuhl and Seeger, 2004).

TIR-FCS with PMO has been employed to detect single nucleotide polymorphisms (SNPs) between short, single-stranded DNA oligomers immobilized on microscope slides and fluorescently tagged ones in solution (Ruckstuhl and Krieg, 2005). As hybridization between short DNA strands is weak, high DNA solution concentrations were required to generate detectable amounts of SNPs. However, as described above, high concentrations of fluorescent molecules in solution, especially for weak surface binding, yield untenably high fluorescence background signals. The use of a PMO and subsequent SAF detection restricted fluorescence detection to that generated very close to the surface, where fluorescent, SNP-related oligomers were present.

A related but slightly different and novel objective that can be used to collect fluorescence at supercritical angles has recently been described (Ries et al., 2008b). This objective consists of a parabolic reflector and an embedded aspheric lens. The parabolic reflector serves to invert the fluorescence exiting the objective such that the lowest angle collected lies at the outer edges of the collimated beam. This arrangement facilitates the use of an iris aperture to alter the lower limit of collected angles, thereby allowing one to easily alter the axial confinement and detection volume. This work also provides a model with which to fit supercritical angle-FCS data.

SAF detection has also been used to facilitate the investigation of myosin lever-arm orientation in skeletal muscle fibers (Burghardt et al., 2007). Myosin is known to reach concentrations of $300 \mu \mathrm{M}$ in parts of muscle fibers, complicating single molecule work. Therefore, a combination of evanescent excitation, SAF detection and CCD detector pixel size was used to limit the detection volume to a few attoL. In this way, the behavior of a protein that is subject to intense molecular crowding in situ was examined.

\section{Surface Plasmon Resonance}

The use of metal films and other types of metal constructions such as corrugated structures or isolated islands is an emerging method in fluorescence microscopy with many potentially very interesting applications. Internal reflection at surfaces coated with thin metal films generates evanescent excitation and emission with unique characteristics, and this optical configuration has been recently explored in a technique called SCPE-FCS (Borejdo et al., 2006). As planepolarized light is internally reflected, incident light at the surface plasmon resonance (SPR) 
angle induces resonant excitation of electron oscillations (surface plasmons). This surface plasmon resonance field propagates along the metal/water interface, manifesting itself as a non-propagating, evanescent field in the aqueous medium. The SPR evanescent field thereby excites fluorescent probes only within its reach.

The dipolar emission field of an individual fluorescent molecule is the superposition of propagating transverse waves in the far-field and non-propagating longitudinal waves (evanescent) in the near-field. In conventional TIRFM, the far-field emission profile is detected. However, in SPR-modified TIRFM, only the near-field is detected when reflections and refractions at the surface convert non-propagating waves to propagating transverse waves. This process is known as surface plasmon coupled emission (SPCE). Converted near-field waves appear at angles greater than the critical angle in the high refractive index medium and therefore, are also supercritical angle fluorescence. The conversion of near-field nonpropagating waves increases with increasing proximity to the interface, providing surface selectivity.

Internally reflecting a light beam at the correct angle, at a surface containing layered glass/ metal/water, has the characteristic that the evanescent wave decays with a shorter characteristic distance. In addition, the metal film quenches fluorescent molecules very close to the surface. These types of surfaces have been combined with FCS in a method called surface plasmon coupled emission fluorescence correlation spectroscopy (SPCE-FCS) (Borejdo et al., 2006). The method was demonstrated by measuring the diffusion of rhodamine-labeled microspheres (100 nm diameter) close to the surfaces. This work demonstrated that with a $48 \mathrm{~nm}$ gold or a $52 \mathrm{~nm}$ silver film, axial excitation was confined to a depth of about $35 \mathrm{~nm}$ or $50 \mathrm{~nm}$, respectively. For these surfaces, the characteristic distances for quenching were $10 \mathrm{~nm}$ and 15 $\mathrm{nm}$, respectively. This work also contains theoretical expressions for the measured autocorrelation function that accounts for the non-exponential excitation/emission profile arising from the combination of evanescent excitation and surface quenching, and demonstrates that SPCE-FCS has a higher signal-to-noise ratio as compared to conventional TIR-FCS.

SPR-modified TIRFM was extended to cellular applications when the technique was used to image rhodamine-labeled cross-bridges in muscle fibers (Burghardt et al., 2006a). Although rhodamine (5' IATR) preferentially labels myosin II in the cross-bridges, actin and $\alpha$-actinin in the z-disk of muscle fibers are also labeled, albeit to a much lesser extent. Nevertheless, the spatial resolution provided by SPR-modified TIRFM was such that it was possible to visualize the z-disk, in addition to the labeled cross-bridges. Comparison with conventional TIRFM also showed that SPR-modified TIRFM reduced background fluorescence and increased axial confinement.

\section{Zero-Mode Waveguides}

Zero-mode waveguides (ZMW) are nanostructures in thin metal films that combine surface plasmons and evanescent illumination to achieve axial confinement. ZMW that consist of subwavelength holes in thin metal films disallow propagating modes above a cut-off wavelength determined by the size and shape of the nanostructure. This effect results in a non-propagating, evanescent field that is attenuated at depths of tens of nanometers, giving detection volumes in the zeptoL $\left(10^{-21} \mathrm{~L}\right)$ range.

Arrays of such waveguides have been used to study DNA polymerase activity at the single molecule level (Levene et al., 2003). Arrays of ZMW were created by etching sub-wavelength holes into $89 \mathrm{~nm}$ thick aluminum films deposited on fused silica substrates. Thereafter, T7 DNA polymerase was immobilized in ZMW such that a single waveguide had at most one polymerase. The efficient and processive synthesis of a DNA strand was observed by following 
the fluorescence emitted by the fluorescently-labeled nucleotide, coumarin-dCTP, which was present at a concentration of $7.5 \mu \mathrm{M}$.

ZMW have been used in combination with FCS to study the oligomerization of the bacteriophage $\lambda$ repressor protein, CI (Samiee et al., 2005). While CI can form dimers, tetramers and octamers, the higher order oligomers are only observed at protein concentrations in the micromolar range. Hence, a $1 \mu \mathrm{M}$ solution of fluorescently-labeled $\mathrm{CI}$ was observed in ZMW, which served to reduce the detection volume and facilitate single-molecule detection. As dimers and tetramers are the dominant species at a concentration of $1 \mu \mathrm{M}$, the fluorescence obtained was autocorrelated and fit to a model assuming one-dimensional diffusion of two species. The parameters obtained from this analysis were used to determine the binding constant for the formation of the CI tetramer. Thus, ZMW in combination with FCS can be used to study biological phenomena by measuring changes in diffusion constants that accompany binding events.

Studies have also shown that it is possible to extend ZMW to cellular applications (MoranMirabal et al., 2007). RBL mast cells were plated on thin gold films (10 nm) etched with ZMW. Scanning electron microscopy (SEM) showed that in regions of membranous extensions (filopodia and lamellipodia) the cell membrane had a tendency to dip down, suggesting that in these places the cell protruded into the nanostructures. To verify this observation, cells labeled with the membrane dye, diIC $_{12}$, were allowed to adhere to the nanofabricated metal surface. The resulting fluorescence, when compared to that of probes outside the sub-wavelength apertures, confirmed that the cells did indeed extend into the ZMW. Furthermore, this process of occupying the nanostructures was an active process requiring actin reorganization. In addition, FCS was used to measure the diffusion coefficients of two membrane dyes, diIC 12 and diIC $_{16}$, in the plasma membranes of the RBL mast cells.

While the ZMW discussed above were circular in shape, rectangular waveguides milled in metal films have also been reported (Wenger et al., 2005). An interesting property of these ZMWs is that, because a rectangular waveguide's cut-off wavelength differs with each axis, the effective site of the detection volume can be tuned by changing the polarization of the incident light relative to the rectangular axes. The volume changes were demonstrated by using diffusional FCS.

\section{Metallic Devices: Fluorescence enhancement and lifetime reduction}

In addition to limiting detection volumes, metallic devices, in particular silver island films (SIF) and some ZMW, can also serve to enhance fluorescence emission (Muthu et al., 2007; Rigneault et al., 2005; Wenger et al., 2005). The quantum yield (Q) of fluorophores is determined by the nonradiative $\left(\mathrm{k}_{\mathrm{nr}}\right)$ and radiative $(\Gamma)$ rate constants as $Q=\Gamma /\left(\Gamma+k_{n r}\right)$. When fluorophores fluoresce in close proximity to SIF surfaces, the radiative rate constant is increased (Lakowicz et al., 2003). This effect serves to increase the quantum yield of the fluorophore, and thereby, the fluorescence intensity.

In the presence of SIFs and some ZMWs lower excitation powers yield the same fluorescence intensities as higher excitation powers in the absence of the metal constructions (Muthu et al., 2007; Rigneault et al., 2005; Wenger et al., 2005). The lower excitation powers help to guard against excessive photobleaching. Furthermore, an increase in the radiative rate constant also serves to decrease the fluorescence lifetime, given by $\tau=1 /\left(\Gamma+k_{n r}\right)$. The shorter fluorescence lifetime decreases the likelihood of a fluorophore reacting with a reactive oxygen species while in the excited state, thereby minimizing photobleaching.

In one experimental demonstration of these effects, myofibrils labeled with rhodaminephalloidin were immobilized on SIF-coated microscope slides (Muthu et al., 2007). The 
myofibrils were illuminated via total internal reflection, and fluorescence images and lifetimes were recorded. Comparison with myofibrils immobilized on bare-glass slides showed that the fluorescence intensities were increased and lifetimes decreased in the presence of SIF.

\section{FUTURE DIRECTIONS}

TIR-FCS is particularly applicable to studies involving planar supported phospholipid membranes because of its surface selectively, requirement for low amounts of material, and sensitivity. The required instrumentation is relatively simple compared to the many more complex but related methods in fluorescence microscopy and should therefore be widely available to many laboratories. As described above, applications of TIR-FCS to supported phospholipid membranes have thus far been limited for the most part to only a small number of studies, including measurement of lipid diffusion in planar membranes (Kannan et al., 2007), characterization of protein and vesicle diffusion very close to supported planar membranes (Starr and Thompson, 2002; Pero et al., 2006; Kyoung and Sheets, 2008) and a single study characterizating specific receptor-ligand interaction kinetics (Lieto et al., 2003). A recent paper describes the experimental details of TIR-FCS in depth, including many of the current pitfalls and limitations, and may serve as a guide for future improvements of the method as applied to supported membranes (Thompson and Steele, 2007).

An avenue possibly worth pursuing with respect to TIR-FCS and supported membranes is to address the kinetics of surface binding when the mechanism is more complex than a simple bimolecular reaction between fluorescent molecules in solution and surface binding sites. One particular case of interest is the one in which a fluorescent species in solution competes with a different, non-fluorescent species in solution for the same surface binding sites. In this case, because of cross-talk, the autocorrelation function contains information about the kinetic association and dissociation rates of the non-fluorescent ligands. Thus, this approach might be developed into a kinetic screen for non-fluorescent ligands. Other investigations involving more complex interaction mechanisms (i.e., those involving multiple reactants) should also be amenable to investigation by TIR-FCS in combination with supported membranes.

Thus far, most TIR-FCS measurements have relied solely on the first-order fluorescence fluctuation autocorrelation function defined in Eq. (1). However, there are a number of alternative and more sophisticated methods for analyzing fluorescence fluctuations, many of which have been used in solution-based FCS, which may prove to be advantageous for TIRFCS studies. One method for analyzing fluorescence fluctuations which is particularly sensitive to molecular clustering is to calculate high-order fluorescence fluctuation autocorrelation functions, defined as

$$
G(\tau)=\frac{<\delta F^{m}(t) \delta F^{n}(t+\tau)>}{<F>^{m+n}}
$$

where $\mathrm{m}$ and/or $\mathrm{n}$ are integers greater than one. This method has been used previously to characterize polydisperse solutions (Palmer and Thompson, 1989a,b;Palmer and Thompson, 1987; Sergeev et al., 2006) but has not yet been extensively explored with respect to evanescent excitation.

A significant, current limitation to understanding biochemical molecular mechanisms which involve multiple components is that characterization of these mechanisms using conventional methods often requires not only the generation and purification of large amounts of material but also unmanageable time expenses. This bottleneck might potentially be overcome by using a combination of nanofabricated microfluidic devices, fast EMCCD cameras, global data analysis, and TIR-FCS. The idea is to create supported membranes containing density gradients 
of reconstituted receptors in one direction and concentration gradients of soluble factors in the other direction by use of microfludic means. Microfluidic chambers have previously been used both with FCS (e.g., Foquet et al., 2004; Foquet et al., 2002; Dittrich and Schwille, 2002) and with TIRFM (Jin et al., 2004; Yang et al., 2003; Yang et al., 2001; Jakeway and de Mello, 2001). In this imagined configuration, fluorescence fluctuation correlation functions calculated in a pixel-dependent manner could at least in theory provide a full spectrum of the surface association and dissociation kinetic rates as a function of the receptor density as well as the solution concentrations of soluble reactants. In addition, using this combination of approaches, it might be possible to characterize the role of solvent flow in biochemical mechanisms thought to be influenced by alignment of reactive species in solution in response to flow.

At least one previous work has begun to explore the use of fast EMCCD cameras with evanescent illumination (Kannan et al., 2007). In this work, one of the primary emphases was on determining how many pixels should separate those from which fluorescence fluctuation autocorrelation functions are calculated in parallel. In the future, examining the full spatiotemporal correlation function promises to provide much more information. This approach has already been demonstrated by using conventional epi-illumination (e.g., Hebert et al., 2005).

A number of TIR-FCS variants, as described above, hold considerable promise if applied to supported membranes. These include TIR-FCS with cross-correlation between species labeled with spectrally resolvable fluorescent probes (Leutenegger et al., 2006); the use of thin metal films or other types of metal constructions (Borejdo et al., 2006; Blom et al, 2006; Burghardt et al. 2006a; Aslan et al., 2005; Maliwal et al., 2003; Lakowicz et al., 2004; Lakowicz, 2001); the use of high refractive index substrates to create very thin evanescent waves (Starr and Thompson, 2000; Borejdo et al. 2006; Muthu et al., 2006); the use of two-photon excitation, either to reduce background from scattered light or for excitation of two different fluorophores with a single light source (Oheim and Schapper, 2005; Schapper et al., 2003; Heinze et al., 2000; Gryczynski et al., 1997; Huang and Thompson, 1993); and the use of the unusual evanescent field polarization (Thompson et al. 1984). Related methods such as near-field scanning optical microscopy (Lewis et al., 2007; Krishnan et al., 2001), focused internal reflection (Burghardt et al., 2006b; Ruckstuhl and Seeger, 2004; Ruckstuhl and Seeger, 2003), super critical angle fluorescence (Axelrod, 2001; Ruckstuhl and Verdes, 2004; Ruckstuhl and Seeger, 2004) and zero mode waveguides (Levene et al., 2003) also hold particular promise for investigation of processess occurring on or near supported membranes when combined with FCS.

Although measurements employing supported membranes are a valuable guide and offer the opportunity of proposing biologically plausible hypotheses resulting from the compositional control available in these studies, ultimately, it is necessary to confirm or dispute these hypotheses based on live cell studies. Abundant possibilities present themselves in this regard. First, when adherent cells are illuminated by evanescent light, only fluorescent molecules bound or close to the basal membrane are excited (Axelrod, 1981). Thus, if intracellular fluorescent molecules are reversibly associating with the cytoplasmic face of the plasma membrane, the kinetics of this process can be characterized by TIR-FCS (Ohsugi et al., 2006; Ohsugi and Kinjo, 2006). Similar measurements have been carried out either by combining evanescent illumination with fluorescence recovery after photobleaching (Sund and Axelrod, 2000) or by imaging single fluorescent molecules as they bind to and dissociate from the cytoplasmic membrane leaflet (Mashanov et al., 2004; Ueda et al., 2001). When combined with an imaging format, this type of measurement produces a kinetic map of the basal cell membrane. Second, for very flat, adherent cells, the evanescent wave might penetrate through the entire cell and illuminate the exterior face of the apical plasma membrane. This arrangement 
would allow use of TIR-FCS to examine the behavior of fluorescent molecules in solution which reversibly interact with sites on the cell exterior.

\section{Acknowledgments}

We thank Bridgett L. Steele and Jamie K. Pero for their contributions. This work was supported by NSF grant MCB-0641087 and NIH grant GM-041402.

\section{REFERENCES}

Agudin JL, Platzeck AM. Fermat's principle and evanescent waves. J. Opts 1978;9:101-106.

Anhut T, Hassler K, Lasser T, Konig K, Rigler R. Fluorescence correlation spectroscopy on dielectric surfaces in total internal reflection geometries. Proc. SPIE 2005;5699:159-166.

Aslan K, Gryczynski I, Malicka J, Matveeva E, Lakowicz JR, Geddes CD. Metal-enhanced fluorescence: an emerging tool in biotechnology. Curr. Opin. Biotech 2005;16:55-62. [PubMed: 15722016]

Axelrod D. Cell-substrate contacts illuminated by total internal reflection fluorescence. J. Cell Biol 1981;89:141-145. [PubMed: 7014571]

Axelrod D, Burghardt TP, Thompson NL. Total internal reflection fluorescence. Annu. Rev. of Biophys. and Bioeng 1984;13:247-268. [PubMed: 6378070]

Axelrod D. Total internal reflection fluorescence microscopy in cell biology. Traffic 2001;2:764-774. [PubMed: 11733042]

Axelrod D. Total internal reflection fluorescence microscopy in cell biology. Meth. Enzymol 2003;361:133. [PubMed: 12624904]

Bacia K, Kim SA, Schwille P. Fluorescence cross-correlation spectroscopy in living cells. Nature Meth 2006;3:83-89.

Bacia K, Schwille P. Practical guidelines for dual-color fluorescence cross-correlation spectroscopy. Nature Protocols 2007;2:2842-2856.

Blom H, Kastrup L, Eggeling C. Fluorescence fluctuation spectroscopy in reduced detection volumes. Curr. Pharm. Biotechno 2006;7:51-66.

Borejdo J, Calander N, Grycznski Z, Grycznski I. Fluorescence correlation spectroscopy in surface plasmon coupled emission microscope. Opt. Exp 2006;14:7878-7888.

Burghardt TP, Thompson NL. Evanescent intensity of a focused Gaussian light beam undergoing total internal reflection in a prism. Opt. Eng 1984;23:62-67.

Burghardt TP, Charlesworth JE, Halstead MF, Tarara JE, Ajtai K. In situ fluorescent protein imaging with metal film-enhanced total internal reflection microscopy. Biophys. J 2006a;90:4662-4671. [PubMed: 16565065]

Burghardt TP, Ajtai K, Borejdo J. In situ single-molecule imaging with attoliter detection using objective total internal reflection confocal microscopy. Biochem 2006b;45:4058-4068. [PubMed: 16566579]

Burghardt TP, Ajtai K, Chan DK, Halstead MF, Li J, Zheng Y. GFP-tagged regulatory light chain monitors single myosin lever-arm orientation in a muscle fiber. Biophys. J 2007;93:2226-2239. [PubMed: 17513376]

Castellana ET, Cremer PS. Solid supported lipid bilayers: From biophysical studies to sensor design. Surf. Sci. Rep 2006;61:429-444.

Chan YHM, Boxer SG. Model membrane systems and their applications. Curr. Opin. Chem. Biol 2007;11:581-587. [PubMed: 17976391]

Costantino S, Comeau JWD, Kolin DL, Wiseman PW. Accuracy and dynamic range of spatial image correlation and cross-correlation spectroscopy. Biophys. J 2005;89:1251-1260. [PubMed: 15923223]

Digman MA, Brown CM, Horwitz AR, Mantulin WW, Gratton E. Paxillin dynamics measured during adhesion assembly and disassembly by correlation spectroscopy. Biophys. J 2008;94:2819-2831. [PubMed: 17993500]

Dittrich PS, Schwille P. Spatial two-photon fluorescence cross-correlation spectroscopy for controlling molecular transport in microfluidic structures. Anal. Chem 2002;74:4472-4479. [PubMed: 12236358] 
Enderlein J, Gregor I, Patra D, Fitter J. Art and artifacts of fluorescence correlation spectroscopy. Curr. Pharm. Biotech 2004;5:155-161.

Foquet M, Korlach J, Zipfel WR, Webb WW, Craighead HG. DNA fragment sizing by single molecule detection in submicrometer-sized closed fluidic channels. Anal. Chem 2002;74:1415-1422. [PubMed: 11922312]

Foquet M, Korlach J, Zipfel WR, Webb WW, Craighead HG. Focal volume confinement by submicrometer-sized fluidic channels. Anal. Chem 2004;76:1618-1626. [PubMed: 15018559]

Garciá-Sáez AJ, Schwille P. Single molecule techniques for the study of membrane proteins. Appl. Mirobiol. Miotechnol 2007;76:257-266.

Girard C, Joachim C, Gauthier S. The physics of the near-field. Rep. Progress Phys 2000;63:893-938.

Gosch M, Rigler R. Fluorescence correlation spectroscopy of molecular motions and kinetics. Adv. Drug Deliv. Rev 2005;57:169-190. [PubMed: 15518928]

Groves JT, Parthasarathy R, Forstner MB. Fluorescence imaging of membrane dynamics. Annu. Rev. Biomed. Eng 2008;10:311-338. [PubMed: 18429702]

Gryczynski I, Gryczynski Z, Lakowicz JR. Two-photon excitation by the evanescent wave from total internal reflection. Anal. Biochem 1997;247:69-76. [PubMed: 9126373]

Hansen RL, Harris JM. Total internal reflection fluorescence correlation spectroscopy for counting molecules at solid/liquid interfaces. Anal. Chem 1998a;70:2565-2575.

Hansen RL, Harris JM. Measuring reversible adsorption kinetics of small molecules at solid/liquid interfaces by total internal reflection fluorescence correlation spectroscopy. Anal. Chem 1998b; 70:4247-4256.

Harlepp S, Robert J, Darnton NC, Chatenay D. Subnanometric measurements of evanescent wave penetration depth using total internal reflection microscopy combined with fluorescent correlation spectroscopy. Appl. Phys. Lett 2004;85:3917-3919.

Hassler K, Anhut T, Rigler R, Gösch M, Lasser T. High count rates with total internal reflection fluorescence correlation spectroscopy. Biophys. J 2005a;88:L01-L03. [PubMed: 15531630]

Hassler K, Leutenegger M, Rigler P, Rao R, Rigler R, Gösch M, Lasser T. Total internal reflection fluorescence correlation spectroscopy (TIR-FCS) with low background and high count-rate per molecule. Opt. Exp 2005b;13:7415-7423.

Hassler K, Rigler P, Blom H, Rigler R, Widengren J, Lasser T. Dynamic disorder in horseradish peroxidase observed with total internal reflection fluorescence correlation spectroscopy. Opt. Exp 2007;15:5366-5375.

Haustein E, Schwille P. Fluorescence correlation spectroscopy: Novel variations of an established technique. Annu. Rev. Biophys. Biom 2007;36:151-169.

Hebert B, Costantino S, Wiseman PW. Spatiotemporal image correlation spectroscopy (STICS): Theory, verification, and application to protein velocity mapping in living $\mathrm{CHO}$ cells. Biophys. J 2005;88:3601-3614. [PubMed: 15722439]

Heinze KG, Koltermann A, Schwille P. Simultaneous two-photon excitation of distinct labels for dualcolor fluorescence cross-correlation analysis. Proc. Natl. Acad. Sci. U.S.A 2000;97:10377-10382. [PubMed: 10973482]

Hellen EH, Axelrod D. Fluorescence emission at dielectric and metal-film interfaces. J. Opt. Soc. B 1987;4:337-350.

Hirschfeld T, Block MJ. Virometer: real time virus detection and identification in biological fluids. Opt. Eng 1977;16:406-407.

Hirschfeld T, Block MJ, Mueller W. Virometer: an optical instrument for visual observation, measurement, and classification of free viruses. J. Histochem. Cytochem 1977;25:719-723. [PubMed: 70452]

Holt M, Cooke A, Neef A, Lagnado L. High mobility of vesicles supports continuous exocytosis at a ribbon synapse. Curr. Biol 2004;14:173-183. [PubMed: 14761649]

Huang Z, Thompson NL. Theory for two-photon excitation in pattern photobleaching with evanescent illumination. Biophy. Chem 1993;47:241-249. 
Huang Z, Pearce KH, Thompson NL. Translational diffusion of bovine prothrombin fragment 1 weakly bound to supported planar membranes: Measurement by total internal reflection with fluorescence pattern photobleaching recovery. Biophys. J 1994;67:1754-1766. [PubMed: 7819508]

Huang Z, Thompson NL. Imaging fluorescence correlation spectroscopy: Nonuniform IgE distributions on planar membranes. Biophys. J 1996;70:2001-2007. [PubMed: 8785359]

Jaiswal JK, Simon SM. Imaging single events at the cell membrane. Nat. Chem. Biol 2007;3:92-98. [PubMed: 17235347]

Jakeway SC, de Mello AJ. Chip-based refractive index detection using a single point evanescent wave probe guide. Analyst 2001;126:1505-1510.

Jin S, Huang P, Park J, Yoo JY, Breuer KS. Near-surface velocimetry using evanescent wave illumination. Expt. Fluids 2004;37:825-833.

Johns LM, Levitan ES, Shelden EA, Holz RW, Axelrod D. Restriction of secretory granule motion near the plasma membrane of chromaffin cells. J. Cell Biol 2001;153:177-190. [PubMed: 11285284]

Kannan B, Guo L, Sudhaharan T, Ahmed S, Maruyama I, Wohland T. Spatially resolved total internal reflection fluorescence correlation microscopy using an electron multiplying charge-coupled device camera. Anal. Chem 2007;79:4463-4470. [PubMed: 17489557]

Kask P, Gunther R, Axhausen P. Statistical accuracy in fluorescence fluctuation experiments. Eur. Biophys. J 1997;25:163-169.

Kiessling, V.; Domanska, MK.; Murray, D.; Wan, C.; Tamm, LK. Wiley Encyclopedia of Chemical Biology. Vol. Volume 4. Hoboken: John Wiley \& Sons; 2009. Supported lipid bilayers; Development and applications in chemical biology; p. 411-422.

Knoll W. Interfaces and thin films as seen by bound electromagnetic waves. Annu. Rev. Phys. Chem 1998;49:569-638. [PubMed: 15012436]

Kolin DL, Costantino S, Wiseman PW. Sampling effects, noise and photobleaching in temporal image correlation spectroscopy. Biophys. J 2006;90:628-639. [PubMed: 16258048]

Koppel DE. Statistical accuracy in fluorescence correlation spectroscopy. Phys. Rev. A 1974;10:19381945.

Krishnan RV, Varma R, Mayor S. Fluorescence methods to probe nanometer-scale organization of molecules in living cell membranes. J. Fluoresc 2001;11:211-226.

Kyoung M, Sheets ED. Vesicle diffusion close to a membrane: intermembrane interactions measured with fluorescence correlation spectroscopy. Biophys. J 2008;95:5789-5797. [PubMed: 18931261]

Lagerholm BC, Weinreb GE, Jacobson K, Thompson NL. Detecting microdomains in intact cell membranes. Annu. Rev. Phys. Chem 2005;56:309-336. [PubMed: 15796703]

Lakowicz JR. Radiative decay engineering: biophysical and biomedical applications. Anal. Biochem 2001;298:1-24. [PubMed: 11673890]

Lakowicz JR, Malicka J, Gryczynski I, Gryczynski Z, Geddes CD. Radiative decay engineering: the role of photonic mode density in biotechnology. J. Appl. Phys. D 2003;36:R240-R249.

Lakowicz JR, Geddes CD, Gryczynski I, Malicka J, Gryczynski Z, Aslan K, Lukomska J, Matveeva E, Zhang JA, Badugu R, Huang J. Advances in surface-enhanced fluorescence. J. Fluoresc 2004;14:425-441. [PubMed: 15617385]

Levene MJ, Korlach J, Turner SW, Foquet M, Craighead HG, Webb WW. Zero-mode waveguides for single-molecule analysis at high concentrations. Science 2003;299:682-686. [PubMed: 12560545]

Leutenegger M, Blom H, Widengren J, Eggeling C, Gösch M, Leitgeb RA, Lasser T. Dual-color total internal reflection fluorescence cross-correlation spectroscopy. J. Biomed. Opt 2006;11:1-3.

Leutenegger M, Lasser T. Detection efficiency in total internal reflection fluorescence microscopy. Opt. Exp 2008;16:8519-8531.

Lewis A, Kuttner YY, Dekhter R, Polhana M. Fluorescence correlation spectroscopy at $100 \mathrm{nM}$ concentrations using near-field scanning optical microscopic (NSOM) geometries and highly diffracting force sensing fiber probes. Israel J. Chem 2007;47:171-176.

Lieto AM, Cush RC, Thompson NL. Ligand-receptor kinetics measured by total internal reflection with fluorescence correlation spectroscopy. Biophys. J 2003;85:3294-3302. [PubMed: 14581230]

Lieto AM, Thompson NL. Total internal reflection with fluorescence correlation spectroscopy: nonfluorescent competitors. Biophys. J 2004;87:1268-1278. [PubMed: 15298929] 
Maliwal BP, Malicka J, Gryczynski I, Gryczynski Z, Lakowicz JR. Fluorescence properties of labeled proteins near silver colloid surfaces. Biopoly 2003;70:585-594.

Mashanov GI, Tacon D, Peckham M, Molloy JE. The spatial and temporal dynamics of pleckstrin homology domain binding at the plasma membrane measured by imaging single molecules in live mouse myoblasts. J. Biol. Chem 2004;279:15274-15280. [PubMed: 14729907]

Mattheyses AL, Axelrod D. Direct measurement of the evanescent field profile produced by objectivebased total internal reflection. J. Biomed. Opt 2006;11:014006. [PubMed: 16526883]

McCain KS, Harris JM. Total internal reflection fluorescence-correlation spectroscopy study of molecular transport in thin sol-gel films. Anal. Chem 2003;75:3616-3624. [PubMed: 14570217]

McCain KS, Schluesche P, Harris JM. Modifying the adsorption behavior of polyamidoamine dendrimers at silica surfaces investigated by total internal reflection fluorescence correlation spectroscopy. Anal. Chem 2004a;76:930-938. [PubMed: 14961722]

McCain KS, Schluesche P, Harris JM. Poly(amidoamine) dendrimers as nanoscale diffusion probes in sol-gel films investigated by total internal reflection fluorescence spectroscopy. Anal. Chem 2004b; 76:939-946. [PubMed: 14961723]

Meseth U, Wohland T, Rigler R, Vogel H. Resolution of fluorescence correlation measurements. Biophys. J 1999;76:1619-1631. [PubMed: 10049342]

Moran-Mirabal JM, Torres AJ, Samiee KT, Baird BA, Craighead HG. Cell investigation of nanostructures: zero-mode waveguides for plasma membrane studies with single molecule resolution. Nanotechnology 2007;18:195101.

Muller JD, Chen Y, Gratton E. Fluorescence correlation spectroscopy. Biophotonics PT B 2003;361:69_ 92.

Muthu P, Grycznski I, Grycznski Z, Talent J, Akopova I, Jain K, Borejdo J. Decreasing photobleaching by silver island films: application to muscle. Anal. Biochem 2007;366:228-236. [PubMed: 17531183]

Oheim M, Schapper F. Non-linear evanescent-field imaging. J. Physics D 2005;38:R185-R197.

Ohsugi Y, Kinjo M. Analysis of membrane-binding protein mobility in living cells using total internal reflection fluorescence correlation spectroscopy. Biophys. Rev. Lett 2006;1:293-299.

Ohsugi Y, Saito K, Tamura M, Kinjo M. Lateral mobility of membrane-binding proteins in living cells measured by total internal reflection fluorescence correlation spectroscopy. Biophys. J 2006;91:3456-3464. [PubMed: 16891361]

Palmer AG, Thompson NL. Molecular aggregation characterized by high order autocorrelation in fluorescence correlation spectroscopy. Biophys. J 1987;52:257-270. [PubMed: 3663831]

Palmer AG, Thompson NL. Fluorescence correlation spectroscopy for detecting submicroscopic clusters of fluorescent molecules in membranes. Chem. Phys. Lipids 1989a;50:253-270. [PubMed: 2548747]

Palmer AG, Thompson NL. High-order fluorescence fluctuation analysis of model protein clusters. Proc. Natl. Acad. Sci. U.S.A 1989b;86:6148-6152. [PubMed: 2548201]

Pero JK, Hass EM, Thompson NL. Size dependence of protein diffusion very close to membrane surfaces: measurement by total internal reflection with fluorescence correlation spectroscopy. J. Phys. Chem. B 2006;110:10910-10918. [PubMed: 16771344]

Qian H. On the statistics of fluorescence correlation spectroscopy. Biophys. Chem 1990;38:49-57. [PubMed: 2085652]

Rigneault H, Capulade J, Dintinger J, Wenger J, Bonod N, Popov E. Enhancement of sinlge-molecular fluorescence detection in subwavelength apertures. PRL 2005;95:117401-1-117401-4.

Ries J, Schwille P. New concepts for fluorescence correlation spectroscopy on membranes. Phys. Chem. Chem. Phys 2008;10:3487-3497. [PubMed: 18548154]

Ries J, Petrov EP, Schwille P. Total internal reflection fluorescence correlation spectroscopy: Effects of lateral diffusion and surface-generated fluorescence. Biophys. J 2008a;95:390-399. [PubMed: 18339763]

Ries J, Ruckstuhl T, Verdes D, Schwille P. Supercritical angle fluorescence correlation spectroscopy. Biophys. J 2008b;94:221-229. [PubMed: 17827221]

Rigler, R.; Elson, EL. Fluorescence correlation spectroscopy: theory and applications. Berlin: Springer; 2001. 
Ruckstuhl T, Seeger S. Confocal total-internal-reflection fluorescence microscopy with a high-aperture parabolic mirror lens. Appl. Optics 2003;42:3277-3283.

Ruckstuhl T, Seeger S. Attoliter detection volumes by confocal total-internal-reflection fluorescence microscopy. Opt. Lett 2004;29:569-571. [PubMed: 15035473]

Ruckstuhl T, Verdes D. Supercritical angle fluorescence (SAF) microscopy. Opt. Exp 2004;12:42464254.

Ruckstuhl T, Krieg A. Microscope objective for large-angle fluorescence used for rapid detection of single nucleotide polymorphisms in DNA hybridization. Anal. Chem 2005;77:2656-2661. [PubMed: 15828807]

Saffarian S, Elson EL. Statistical analysis of fluorescence correlation spectroscopy: The standard deviation and bias. Biophys. J 2003;84:2030-2042. [PubMed: 12609905]

Samiee KT, Foquet M, Guo L, Cox EC, Craighead HG. $\lambda$-Repressor oligomerization kinetics at high concentrations using fluorescence correlation spectroscopy in zero-mode waveguides. Biophys. $\mathrm{J}$ 2005;88:2145-2153. [PubMed: 15613638]

Schapper F, Goncalves JT, Oheim M. Fluorescence imaging with two-photon evanescent wave excitation. Eur. Biophys. J 2003;32:635-643. [PubMed: 12955359]

Sergeev M, Costantino S, Wiseman PW. Measurement of monomer-oligomer distributions via fluorescence moment image analysis. Biophys. J 2006;91:3884-3896. [PubMed: 16935950]

Schneckenburger H. Total internal reflection fluorescence microscopy: technical innovations and novel applications. Curr. Opin. Biotech 2005;16:13-18. [PubMed: 15722010]

Schwille P, Meyer-Almes FJ, Rigler R. Dual-color fluorescence cross-correlation spectroscopy for multicomponent diffusional analysis in solution. Biophys. J 1997;72:1878-1886. [PubMed: 9083691]

Sonesson AW, Blom H, Hassler K, Elofsson UM, Callisen TH, Widengren J, Brismar H. Proteinsurfactant interactions at hydrophobic interfaces studied with total internal reflection fluorescence correlation spectroscopy (TRI-FCS). J. Colloid Interf. Sci 2008;317:449-457.

Starr TE, Thompson NL. Formation and characterization of planar phospholipid bilayers supported on TiO2 and SrTiO3 single crystals. Langmuir 2000;16:10301-10308.

Starr TE, Thompson NL. Total internal reflection with fluorescence correlation spectroscopy: combined surface reaction and solution diffusion. Biophys. J 2001;80:1575-1584. [PubMed: 11222318]

Starr TE, Thompson NL. Local diffusion and concentration of IgG near planar membranes: measurement by total internal reflection with fluorescence correlation spectroscopy. J. Phys. Chem. B 2002;106:2365-2371.

Steyer JA, Almers W. A real-time view of life within $100 \mathrm{~nm}$ of the plasma membrane. Nat. Rev. Mol. Cell Biol 2001;2:268-275. [PubMed: 11283724]

Sund SE, Axelrod D. Actin dynamics at the living cell submembrane imaged by total internal reflection fluorescence photobleaching. Biophys. J 2000;79:1655-1669. [PubMed: 10969025]

Thompson NL, Burghardt TP, Axelrod D. Measuring surface dynamics of biomolecules by total internal reflection fluorescence with photobleaching recovery or correlation spectroscopy. Biophys. J 1981;33:435-454. [PubMed: 7225515]

Thompson NL. Surface binding rates of nonfluorescent molecules may be obtained by total internal reflection with fluorescence correlation spectroscopy. Biophys. J 1982;38:327-329. [PubMed: 7104443]

Thompson NL, Axelrod D. Immunoglobulin surface-binding kinetics studied by total internal reflection with fluorescence correlation spectroscopy. Biophys. J 1983;43:103-114. [PubMed: 6882857]

Thompson NL, McConnell HM, Burghardt TP. Order in supported phospholipid monolayers detected by the dichroism of fluorescence excited by polarized evanescent illumination. Biophys. $\mathrm{J}$ 1984;46:739-747. [PubMed: 6518254]

Thompson, NL. Fluorescence Correlation Spectroscopy. In: Lakowicz, JR., editor. Topics in Fluorescence Spectroscopy. Vol. Vol. 3. New York: Plenum Press; 1991. p. 337-378.

Thompson NL, Pearce KH, Hsieh HV. Total internal reflection fluorescence microscopy: application to substrate-supported planar membranes. Europ. Biophys. J 1993;22:367-378. 
Thompson NL, Lieto AM, Allen NW. Recent advances in fluorescence correlation spectroscopy. Curr. Opin. Struct. Biol 2002;12:634-641. [PubMed: 12464316]

Thompson, NL.; Pero, JK. Fluorescence Spectroscopy in Biology: Advanced Methods and Their Applications to Membranes, Proteins, DNA and Cells. Berlin: Springer-Verlag; 2005. p. 79-103.

Thompson, NL.; Pero, JK. Reviews in Fluorescence 2006. Vol. Vol.3. New York: Springer; 2006. Total internal reflection-fluorescence correlation spectroscopy; p. 215-237.

Thompson NL, Steele BL. Total internal reflection with fluorescence correlation spectroscopy. Nat. Protoc 2007;2:878-890. [PubMed: 17446873]

Ueda M, Sako Y, Tanaka T, Devreotes P, Yanagida T. Single molecule analysis of chemotactic signaling in Dictyostelium cells. Science 2001;294:864-867. [PubMed: 11679673]

Vukojevic V, Pramanik A, Yakovleva T, Rigler R, Terenius L, Bakalkin G. Study of molecular events in cells by fluorescence correlation spectroscopy. Cell. Molec. Life Sciences 2005;62:535-550.

Wallace MI, Ying LM, Balasubramanian S, Klenerman D. FRET fluctuation spectroscopy: exploring the conformational dynamics of a DNA hairpin loop. J. Phys. Chem. B 2000;104:11551-11555.

Wenger J, Lenne P-F, Popov E, Rigneault H. Single molecule fluorescence in rectangular nano-apertures. Opt. Exp 2005;2005:7035-7044.

Widengren J, Mets U, Rigler R. Fluorescence correlation spectroscopy of triplet states in solution: a theoretical and experimental study. J. Phys. Chem 1995;99:13368-13379.

Widengren J, Schweinberger E, Berger S, Seidel CAM. Two new concepts to measure fluorescence resonance energy transfer via fluorescence correlation spectroscopy: theory and experimental realizations. J. Phys. Chem. A 2001;105:6851-6866.

Wohland T, Rigler R, Vogel H. The standard deviation in fluorescence correlation spectroscopy. Biophys. J 2001;80:2987-2999. [PubMed: 11371471]

Yang TL, Baryshnikova OK, Mao HB, Holden MA, Cremer PS. Investigations of bivalent antibody binding on fluid-supported phospholipid membranes: the effect of hapten density. J. Amer. Chem. Soc 2003;125:4779 -4784. [PubMed: 12696896]

Yang TL, Jung SY, Mao HB, Cremer PS. Fabrication of phospholipid bilayer coated microchannels for on-chip immunoassays. Anal. Chem 2001;73:165-169. [PubMed: 11199961] 
(a)

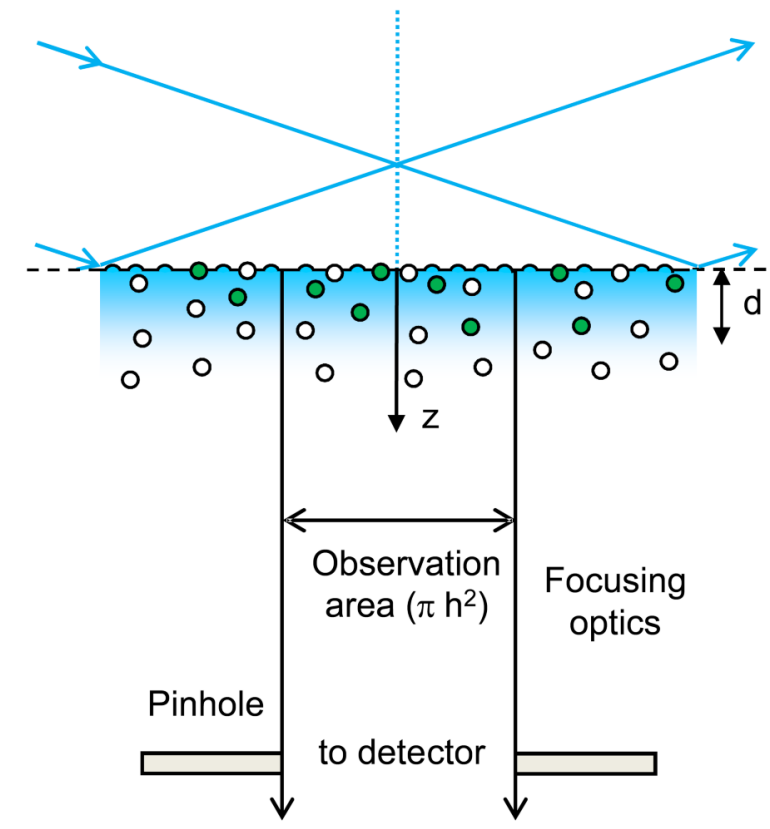

(c)
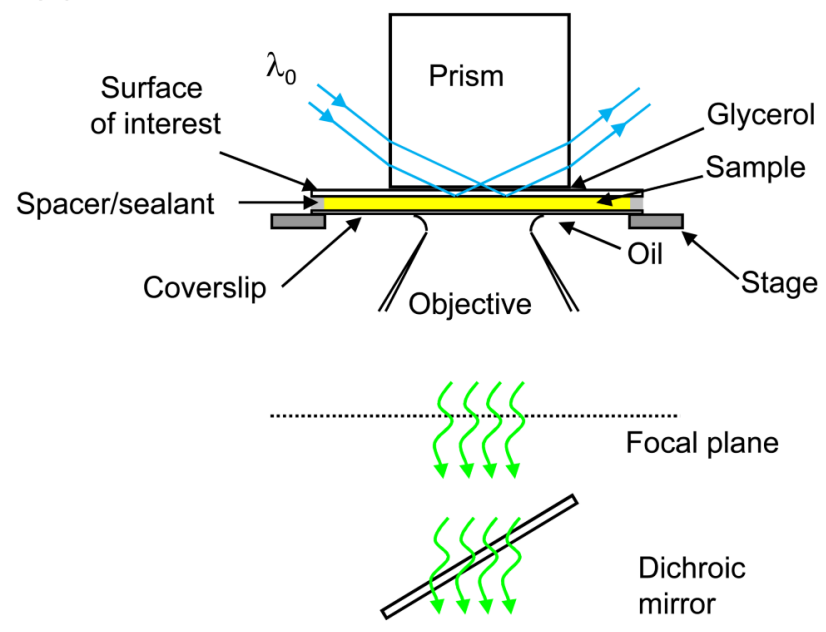

Dichroic mirror (b)

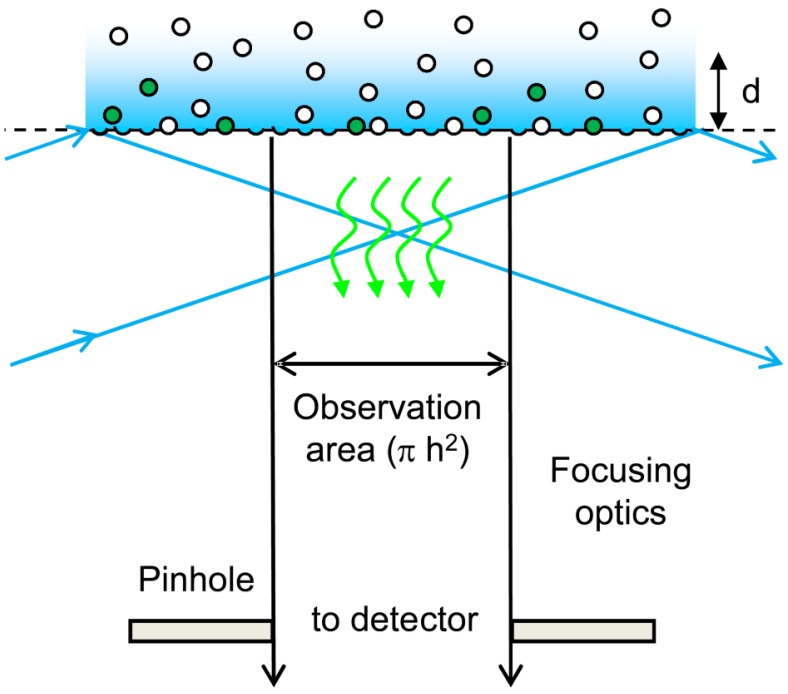

(d)

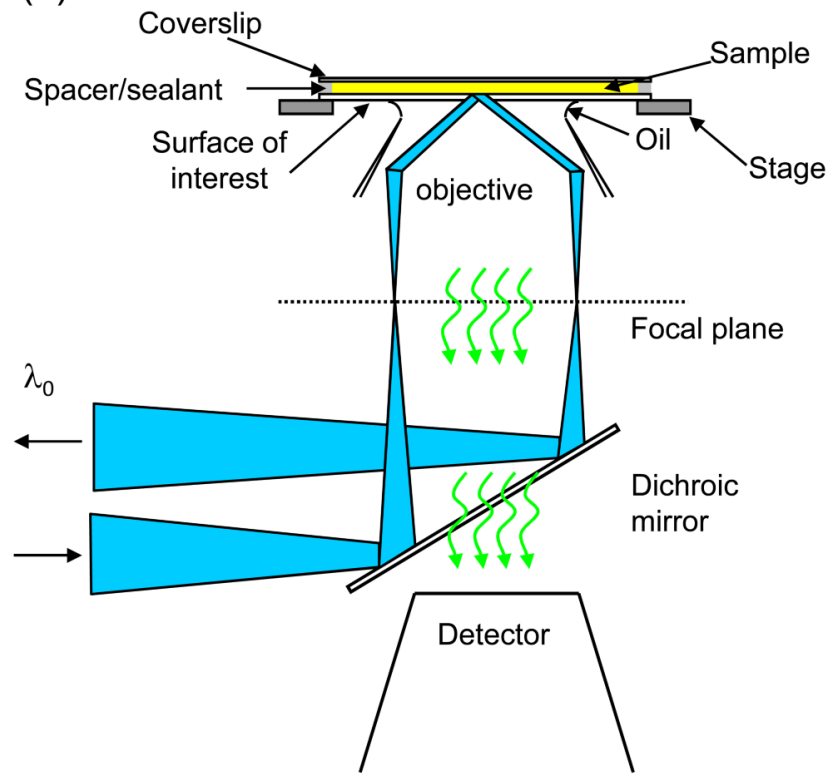

Figure 1. Through-Prism and Through-Objective TIR-FCS

$(a, c)$ In through-prism TIR-FCS, a laser beam is totally internally reflected into a sample through a prism that is mounted on the stage of an inverted microscope. (b,d) In throughobjective TIR-FCS, a laser beam is directed through a microscope port, reflected towards the sample plane with a dichroic mirror, and is focused through the periphery of a high numerical aperture objective so that the beam internally reflects at the sample plane. In all cases, the evanescently excited fluorescence is collected through the high-aperture objective and passed through a spectral filter to remove scattered evanescent light. The light is passed through a small aperture or collected with a fiber optic, placed at a back image plane of the microscope, 
before reaching a single-photon counting detector. The detector signal is processed using either a computer with a correlator card and associated software or similar in-house constructed signal processing equipment. 
(a)

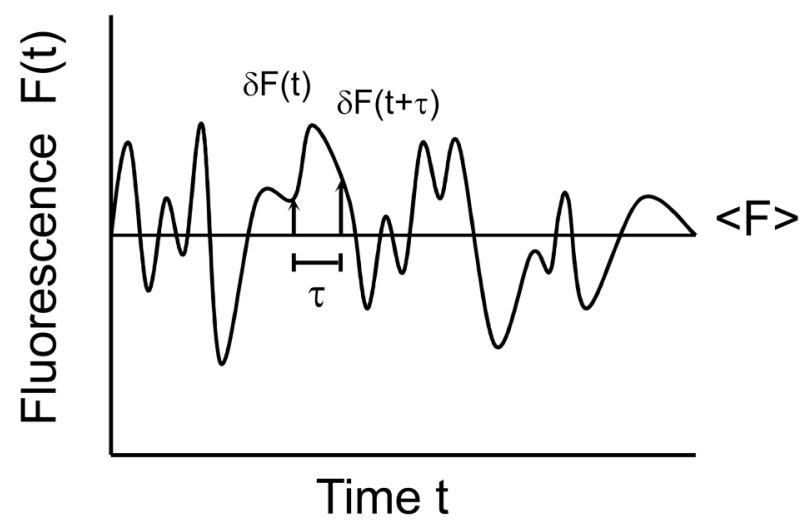

(b)

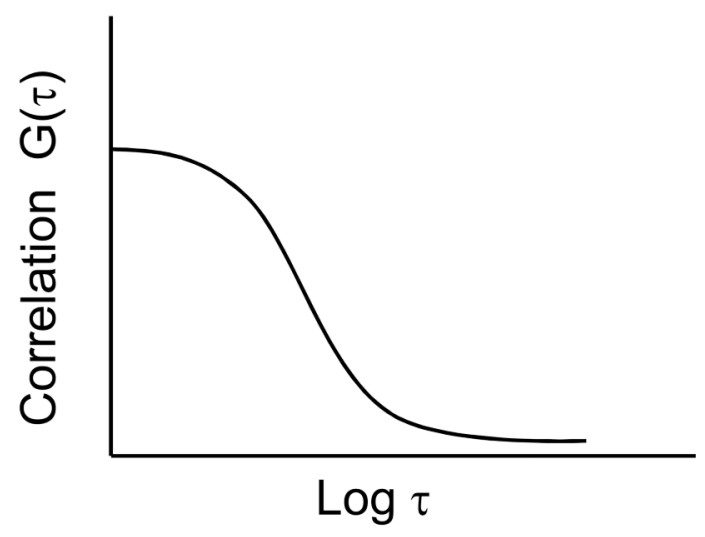

Figure 2. Fluorescence Fluctuation Autocorrelation Functions

(a) The fluorescence measured from the observation volume fluctuates with time as individual fluorescent molecules diffuse through the evanescent field, associate with and dissociate from surface binding sites, and/or undergo transitions between states with different detected fluorescence intensities. (b) The temporal fluorescence fluctuations are autocorrelated (see Eq. $1)$. 
(a)

(b)

\section{Supported planar membrane}

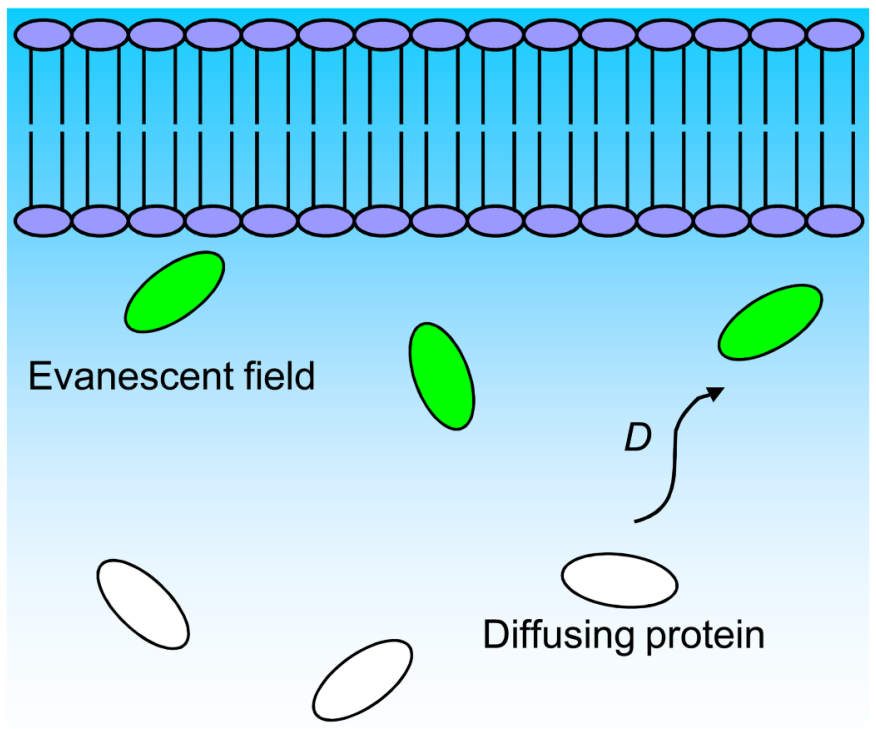

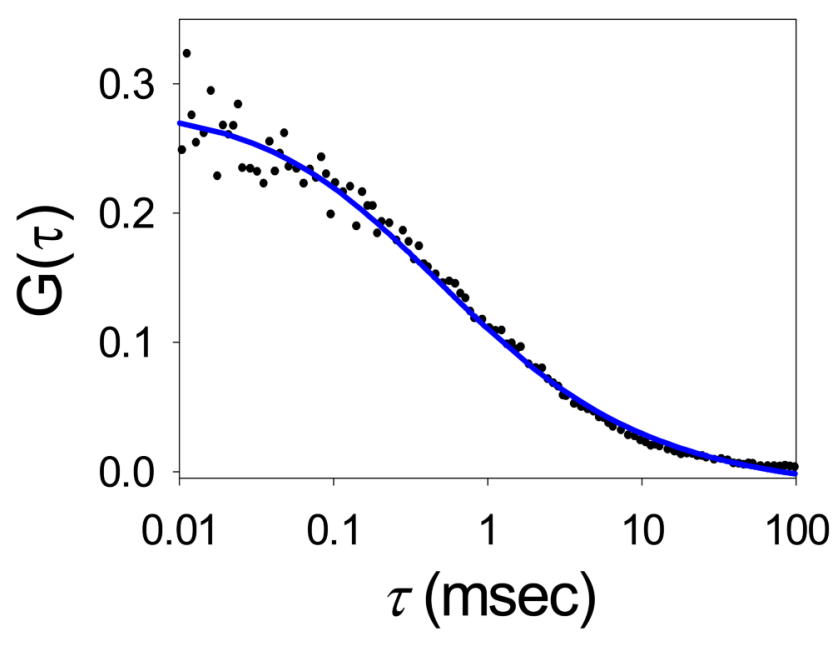

Figure 3. Diffusion Through the Evanescent Wave

(a) Schematic of diffusion close to a supported planar membrane. Fluorophores in the path of the evanescent wave are excited, and fluoresce. (b) Representative TIR-FCS data of diffusion, fit to an appropriate theoretical autocorrelation function. The data pertain to $10 \mathrm{nM}$ Alexa448labeled IgG in PBS with $1 \mathrm{mg} \mathrm{mL}^{-1}$ unlabeled $\mathrm{IgG}$ and $10 \mathrm{mg} \mathrm{mL}^{-1}$ ovalbumin diffusing close to a supported planar membrane. Reproduced with permission from Biophys. J. 2003. 85, 3294-3302. 
(a)

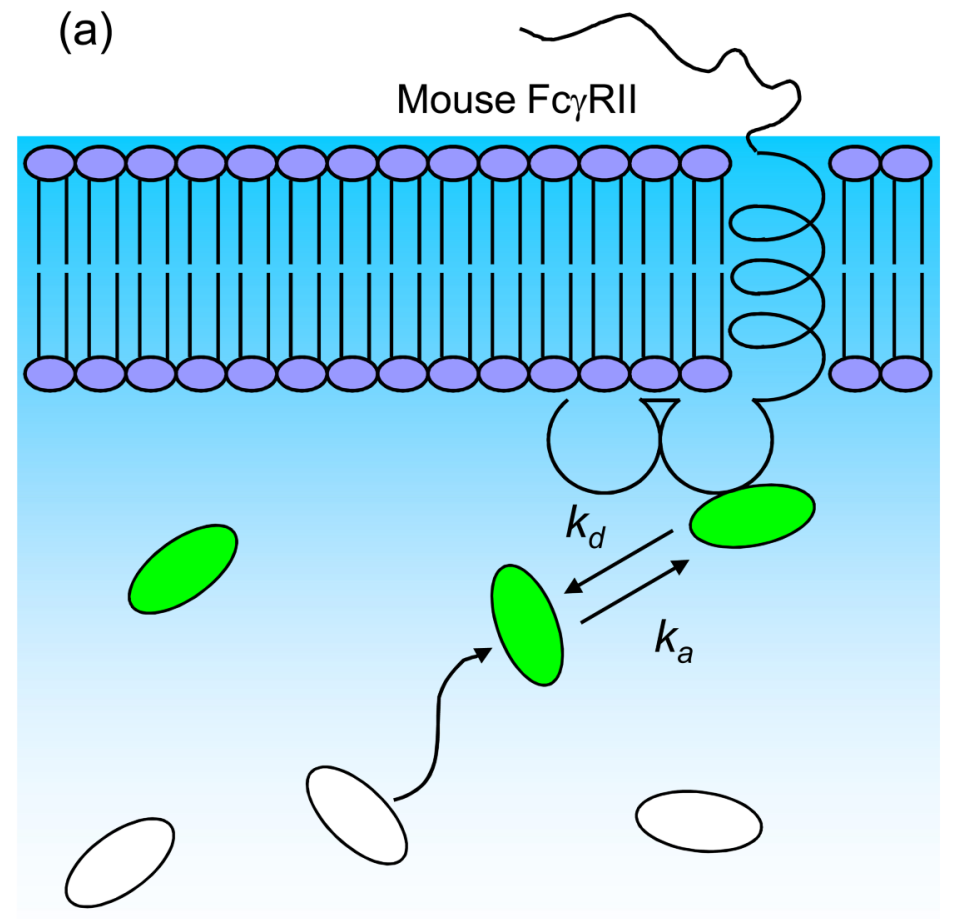

(b)

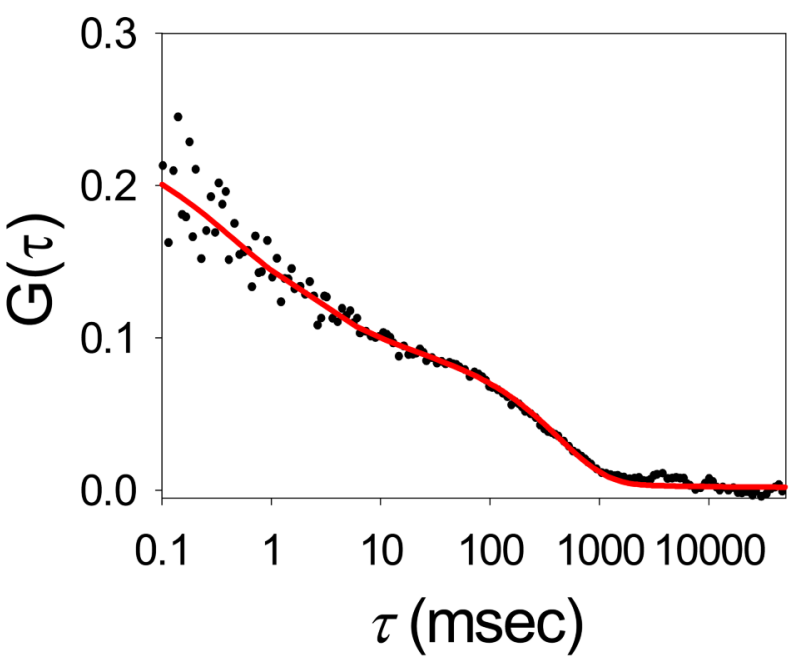

Figure 4. Surface Binding Kinetics

(a) Schematic of soluble ligands reversibly binding at sites on supported planar membranes. In the above figure, $\mathrm{k}_{\mathrm{a}}$ and $\mathrm{k}_{\mathrm{d}}$ are the association and dissociation rate constants of a soluble ligand, IgG, interacting with mouse Fc $\gamma$ RII reconstituted in supported planar membranes. (b) Representative TIR-FCS data of surface binding kinetics, fit to an appropriate theoretical model. The data pertain to $10 \mathrm{nM}$ Alexa448-labeled IgG in PBS, with $1 \mathrm{mg} \mathrm{mL}^{-1}$ unlabeled $\mathrm{IgG}$ and $10 \mathrm{mg} \mathrm{mL}^{-1}$ ovalbumin, interacting with mouse Fc $\gamma \mathrm{RII}$ reconstituted in supported planar membranes. Reproduced with permission from Biophys. J. 2003. 85, 3294-3302. 